\title{
FUZZY STABILITY OF A GENERALIZED QUADRATIC FUNCTIONAL EQUATION
}

\author{
Abbas Najati
}

\begin{abstract}
We prove the generalized Hyers-Ulam stability of the generalized quadratic functional equation

$$
f(r x+s y)=r^{2} f(x)+s^{2} f(y)+\frac{r s}{2}[f(x+y)-f(x-y)]
$$

in fuzzy Banach spaces, where $r, s$ are non-zero rational numbers with $r^{2}+s^{2} \neq 1$.
\end{abstract}

\section{Introduction}

The stability problem of functional equations originated from a question of Ulam [27] concerning the stability of group homomorphisms: Let $\left(G_{1}, *\right)$ be a group and let $\left(G_{2}, \diamond, d\right)$ be a metric group with the metric $d(\cdot, \cdot)$. Given $\epsilon>0$, does there exist $\delta(\epsilon)>0$ such that if a mapping $h: G_{1} \rightarrow G_{2}$ satisfies the inequality

$$
d(h(x * y), h(x) \diamond h(y))<\delta
$$

for all $x, y \in G_{1}$, then there is a homomorphism $H: G_{1} \rightarrow G_{2}$ with

$$
d(h(x), H(x))<\epsilon
$$

for all $x \in G_{1}$ ?

Hyers [11] gave a first affirmative answer to the question of Ulam for Banach spaces. Aoki [3] and Th. M. Rassias [24] provided a generalization of the Hyers' theorem for additive and linear mappings, respectively, by allowing the Cauchy difference to be unbounded. P. Găvruta [9] provided a further generalization of the Th. M. Rassias' theorem by using a general control function.

The functional equation

$$
f(x+y)+f(x-y)=2 f(x)+2 f(y)
$$

is called a quadratic functional equation. Quadratic functional equations were used to characterize inner product spaces $[1,2,13]$. In particular, every solution of the quadratic equation (1.1) is said to be a quadratic mapping. It is well

Received July 21, 2009; Revised November 3, 2009.

2000 Mathematics Subject Classification. Primary 46S40; Secondary 39B52, 39B82, 26E50, 46S50.

Key words and phrases. stability, fuzzy Banach spaces, quadratic mapping. 
known that a mapping $f$ between real vector spaces is quadratic if and only if there exists a unique symmetric bi-additive mapping $B$ such that $f(x)=$ $B(x, x)$ for all $x$ (see $[1,16])$. The bi-additive mapping $B$ is given by

$$
B(x, y)=\frac{1}{4}[f(x+y)-f(x-y)] .
$$

The generalized Hyers-Ulam stability problem for the quadratic functional equation (1.1) was proved by $\mathrm{F}$. Skof for mappings $f: E_{1} \rightarrow E_{2}$, where $E_{1}$ is a normed space and $E_{2}$ is a Banach space (see [26]). Cholewa [6] noticed that the theorem of Skof is still true if the relevant domain $E_{1}$ is replaced by an abelian group. In [7], Czerwik proved the generalized Hyers-Ulam stability of the quadratic functional equation (1.1). Grabiec [10] has generalized these results mentioned above. Jun and Lee [14] proved the generalized Hyers-Ulam stability of a Pexiderized quadratic equation. The stability problems of several functional equations have been extensively investigated by a number of authors and there are many interesting results concerning this problem (see [14]-[23]). We also refer the readers to the books [8], [12], [15] and [25].

We recall some basic facts concerning fuzzy Banach spaces and some preliminary results.

Definition 1.1 ([4]). Let $X$ be a real vector space. A function $N: X \times \mathbb{R} \rightarrow$ $[0,1]$ is called a fuzzy norm on $X$ if for all $x, y \in X$ and all $s, t \in \mathbb{R}$,

$\left(N_{1}\right) N(x, t)=0$ for $t \leq 0$

$\left(N_{2}\right) x=0$ if and only if $N(x, t)=1$ for all $t>0$;

$\left(N_{3}\right) N(c x, t)=N\left(x, \frac{t}{|c|}\right)$ if $c \neq 0$;

$\left(N_{4}\right) N(x+y, s+t) \geq \min \{N(x, s), N(y, t)\}$;

$\left(N_{5}\right) N(x, \cdot)$ is a non-decreasing function of $\mathbb{R}$ and $\lim _{t \rightarrow \infty} N(x, t)=1$;

$\left(N_{6}\right)$ for $x \neq 0, N(x, \cdot)$ is continuous on $\mathbb{R}$.

The pair $(X, N)$ is called a fuzzy normed vector space.

Example 1.2. Let $(X,\|\cdot\|)$ be a normed linear space and $\alpha, \beta>0$. Then

$$
N(x, t)= \begin{cases}\frac{\alpha t}{\alpha t+\beta\|x\|}, & t>0, x \in X \\ 0, & t \leq 0, x \in X\end{cases}
$$

is a fuzzy norm on $X$.

Example 1.3. Let $(X,\|\cdot\|)$ be a normed linear space and $\beta>\alpha>0$. Then

$$
N(x, t)= \begin{cases}0, & t \leq \alpha\|x\| \\ \frac{t}{t+(\beta-\alpha)\|x\|}, & \alpha\|x\|<t \leq \beta\|x\| ; \\ 1, & t>\beta\|x\|\end{cases}
$$

is a fuzzy norm on $X$.

Definition 1.4. Let $(X, N)$ be a fuzzy normed space. A sequence $\left\{x_{n}\right\}$ in $X$ is said to be convergent if there exists $x \in X$ such that $\lim _{n \rightarrow \infty} N\left(x_{n}-x, t\right)=1$ for all $t>0$. In this case, $x$ is called the limit of the sequence $\left\{x_{n}\right\}$ and we denote it by $N-\lim x_{n}=x$. 
The limit of the convergent sequence $\left\{x_{n}\right\}$ in $(X, N)$ is unique. Since if $N$ - $\lim x_{n}=x$ and $N$-lim $x_{n}=y$ for some $x, y \in X$, we have from $\left(N_{4}\right)$ that

$$
N(x-y, t) \geq \min \left\{N\left(x-x_{n}, t / 2\right), N\left(x_{n}-y, t / 2\right)\right\}
$$

for all $t>0$ and all $n \in \mathbb{N}$. So $N(x-y, t)=1$ for all $t>0$. Hence $\left(N_{2}\right)$ implies that $x=y$.

Definition 1.5. Let $(X, N)$ be a fuzzy normed space. A sequence $\left\{x_{n}\right\}$ in $X$ is called Cauchy if for each $\varepsilon>0$ and each $t>0$ there exists $M \in \mathbb{N}$ such that for all $n \geq M$ and all $p>0$, we have $N\left(x_{n+p}-x_{n}, t\right)>1-\varepsilon$.

It follows from $\left(N_{4}\right)$ that every convergent sequence in a fuzzy normed space is Cauchy. If in a fuzzy normed space, each Cauchy sequence is convergent, then the fuzzy norm is said to be complete and the fuzzy normed space is called a fuzzy Banach space.

Example 1.6. Let $N: \mathbb{R} \times \mathbb{R} \rightarrow[0,1]$ be a fuzzy norm on $\mathbb{R}$ defined by

$$
N(x, t)= \begin{cases}\frac{t}{t+|x|}, & t>0 \\ 0, & t \leq 0 .\end{cases}
$$

Then $(\mathbb{R}, N)$ is a fuzzy Banach space. Let $\left\{x_{n}\right\}$ be a Cauchy sequence in $\mathbb{R}$, $\delta>0$ and $\varepsilon=\frac{\delta}{1+\delta}$. Then there exists $M \in \mathbb{N}$ such that for all $n \geq M$ and all $p>0$, we have $\frac{1}{1+\left|x_{n+p}-x_{n}\right|}>1-\varepsilon$. So $\left|x_{n+p}-x_{n}\right|<\delta$ for all $n \geq M$ and all $p>0$. Therefore $\left\{x_{n}\right\}$ is a Cauchy sequence in $(\mathbb{R},|\cdot|)$. Let $x_{n} \rightarrow x_{0} \in \mathbb{R}$ as $n \rightarrow \infty$. Then $\lim _{n \rightarrow \infty} N\left(x_{n}-x_{0}, t\right)=1$ for all $t>0$.

We say that a mapping $f: X \rightarrow Y$ between fuzzy normed vector spaces $X$ and $Y$ is continuous at a point $x_{0} \in X$ if for each sequence $\left\{x_{n}\right\}$ converging to $x_{0}$ in $X$, then the sequence $\left\{f\left(x_{n}\right)\right\}$ converges to $f\left(x_{0}\right)$. If $f: X \rightarrow Y$ is continuous at each $x \in X$, then $f: X \rightarrow Y$ is said to be continuous on $X$. For different types of continuity such as fuzzy continuity, sequential fuzzy continuity, weakly fuzzy continuity and strongly fuzzy continuity of an operator over fuzzy normed linear spaces we refer the interested reader to [5].

Throughout this paper, we assume that $r, s$ are non-zero rational numbers with $r^{2}+s^{2} \neq 1$, and that $X$ is a vector space and that $(Y, N)$ is a fuzzy Banach space.

In this paper, we prove the generalized Hyers-Ulam stability of the following generalized quadratic functional equation

$$
f(r x+s y)=r^{2} f(x)+s^{2} f(y)+\frac{r s}{2}[f(x+y)-f(x-y)]
$$

in fuzzy Banach spaces. Letting $r=s=1$ in (1.2), we get the quadratic functional equation (1.1). For convenience, we use the following abbreviation for a given mapping $f: X \rightarrow Y$,

$$
D f(x, y):=f(r x+s y)-r^{2} f(x)-s^{2} f(y)-\frac{r s}{2}[f(x+y)-f(x-y)]
$$

for all $x, y \in X$. 


\section{Stability of the generalized quadratic functional equation (1.2)}

We start this section with the following result concerning on the functional equation (1.2).

Proposition 2.1 ([21]). Let $\mathcal{X}$ and $\mathcal{Y}$ be real vector spaces. A mapping $f$ : $\mathcal{X} \rightarrow \mathcal{Y}$ satisfies

$$
D f(x, y)=0
$$

for all $x, y \in \mathcal{X}$ if and only if $f$ is quadratic.

Now we prove the generalized Hyers-Ulam stability of the quadratic functional equation (1.2) in fuzzy Banach spaces. In this section $X$ is a linear space, $\mathbb{X}$ is a linear normed space and $(Y, N)$ is a fuzzy Banach space.

Theorem 2.2. Let $\varphi: X^{2} \backslash\{(0,0)\} \rightarrow(0, \infty)$ be a function such that

$$
\widetilde{\varphi}(x, y):=\sum_{n=0}^{\infty} \frac{\varphi\left(r^{n} x, r^{n} y\right)}{r^{2 n}}<\infty
$$

for all $(x, y) \in X^{2} \backslash\{(0,0)\}$. Let $f: X \rightarrow Y$ be a mapping with $f(0)=0$ such that

$$
\lim _{t \rightarrow \infty} N(D f(x, y), t \varphi(x, y))=1
$$

uniformly on $X^{2} \backslash\{(0,0)\}$. Then $Q(x):=N-\lim _{n \rightarrow \infty} \frac{f\left(r^{n} x\right)}{r^{2 n}}$ exists for each $x \in X$ and defines a quadratic mapping $Q: X \rightarrow Y$ such that if for some $\delta>0, \alpha>0$

$$
N(D f(x, y), \delta \varphi(x, y)) \geq \alpha
$$

for all $(x, y) \in X^{2} \backslash\{(0,0)\}$, then

$$
N\left(f(x)-Q(x), \frac{\delta}{r^{2}} \widetilde{\varphi}(x, 0)\right) \geq \alpha
$$

for all $x \in X \backslash\{0\}$.

Furthermore, the quadratic mapping $Q: X \rightarrow Y$ is a unique mapping such that

$$
\lim _{t \rightarrow \infty} N(f(x)-Q(x), t \widetilde{\varphi}(x, 0))=1
$$

uniformly on $X \backslash\{0\}$.

Proof. For a given $\varepsilon>0$, by (2.3), we can find some $t_{0}>0$ such that

$$
N(D f(x, y), t \varphi(x, y)) \geq 1-\varepsilon
$$

for all $(x, y) \in X^{2} \backslash\{(0,0)\}$ and all $t \geq t_{0}$. Letting $y=0$ in $(2.7)$, we get

$$
N\left(f(r x)-r^{2} f(x), t \varphi(x, 0)\right) \geq 1-\varepsilon
$$


for all $x \in X \backslash\{0\}$ and all $t \geq t_{0}$. Replacing $x$ by $r^{n} x$ in (2.8) and using $\left(N_{3}\right)$, we get

$$
N\left(\frac{f\left(r^{n+1} x\right)}{r^{2 n+2}}-\frac{f\left(r^{n} x\right)}{r^{2 n}}, \frac{t}{r^{2 n+2}} \varphi\left(r^{n} x, 0\right)\right) \geq 1-\varepsilon
$$

for all $x \in X \backslash\{0\}$, all $t \geq t_{0}$ and all integers $n \geq 0$. Since

$$
\begin{aligned}
& N\left(\frac{f\left(r^{n+p} x\right)}{r^{2(n+p)}}-\frac{f\left(r^{n} x\right)}{r^{2 n}}, \sum_{k=n}^{n+p-1} \frac{t}{r^{2 k+2}} \varphi\left(r^{k} x, 0\right)\right) \\
= & N\left(\sum_{k=n}^{n+p-1}\left[\frac{f\left(r^{k+1} x\right)}{r^{2 k+2}}-\frac{f\left(r^{k} x\right)}{r^{2 k}}\right], \sum_{k=n}^{n+p-1} \frac{t}{r^{2 k+2}} \varphi\left(r^{k} x, 0\right)\right) \\
\geq & \min _{n \leq k \leq n+p-1}\left\{N\left(\frac{f\left(r^{k+1} x\right)}{r^{2 k+2}}-\frac{f\left(r^{k} x\right)}{r^{2 k}}, \frac{t}{r^{2 k+2}} \varphi\left(r^{k} x, 0\right)\right)\right\},
\end{aligned}
$$

we get from (2.9) that

$$
N\left(\frac{f\left(r^{n+p} x\right)}{r^{2(n+p)}}-\frac{f\left(r^{n} x\right)}{r^{2 n}}, \sum_{k=n}^{n+p-1} \frac{t_{0}}{r^{2 k+2}} \varphi\left(r^{k} x, 0\right)\right) \geq 1-\varepsilon
$$

for all $x \in X \backslash\{0\}$ and all integers $n \geq 0, p \geq 1$. Let $x \in X \backslash\{0\}$ and $\delta>0$. It follows from (2.2) that there exists $M \in \mathbb{N}$ such that

$$
\frac{t_{0}}{r^{2}} \sum_{k=n}^{n+p-1} \frac{\varphi\left(r^{k} x, 0\right)}{r^{2 k}}<\delta
$$

for all $n \geq M$ and all integers $p \geq 1$. Now we deduce from $\left(N_{5}\right)$ and (2.10) that

$$
N\left(\frac{f\left(r^{n+p} x\right)}{r^{2(n+p)}}-\frac{f\left(r^{n} x\right)}{r^{2 n}}, \delta\right) \geq 1-\varepsilon
$$

for all $n \geq M$ and all integers $p \geq 1$. Hence $\left\{\frac{f\left(r^{n} x\right)}{r^{2 n}}\right\}$ is a Cauchy sequence in $Y$. Since $Y$ is a fuzzy Banach space, this sequence converges to some $Q(x) \in$ $Y$. Since $f(0)=0$, we can define a mapping $Q: X \rightarrow Y$ by $Q(x):=N$ $\lim _{n \rightarrow \infty} \frac{f\left(r^{n} x\right)}{r^{2 n}}$, i.e., for each $t>0$ and $x \in X, \lim _{n \rightarrow \infty} N\left(\frac{f\left(r^{n} x\right)}{r^{2 n}}-Q(x), t\right)=1$.

Let $x, y \in X$ and $t>0$. It follows from $\left(N_{2}\right)$ and $\left(N_{4}\right)$ that

$$
\begin{aligned}
& N(D Q(x, y), t) \\
& \geq \min \{ N\left(Q(r x+s y)-\frac{f\left(r^{n}(r x+s y)\right)}{r^{2 n}}, \frac{t}{5}\right), \\
& N\left(r^{2} \frac{f\left(r^{n} x\right)}{r^{2 n}}-r^{2} Q(x), \frac{t}{5}\right), N\left(s^{2} \frac{f\left(r^{n} y\right)}{r^{2 n}}-s^{2} Q(y), \frac{t}{5}\right), \\
& N\left(\frac{r s\left[f\left(r^{n}(x+y)\right)-f\left(r^{n}(x-y)\right)\right]}{2 r^{2 n}}-\frac{r s}{2}[Q(x+y)-Q(x-y)], \frac{t}{5}\right), \\
&\left.N\left(D f\left(r^{n} x, r^{n} y\right), \frac{r^{2 n} t}{5}\right)\right\}
\end{aligned}
$$


for all $n \geq 0$. Let $0<\varepsilon<1$. From the definition of $Q$, there exists $M_{0} \in \mathbb{N}$ such that the first four terms on the right-hand side of the above inequality are greater than $1-\varepsilon$ for all $n \geq M_{0}$. By (2.3), we can find some $t_{0}>0$ such that (2.7) holds true. Since $\lim _{n \rightarrow \infty} r^{-2 n} \varphi\left(r^{n} x, r^{n} y\right)=0$, there exists $M_{1} \geq M_{0}$ such that $t_{0} \varphi\left(r^{n} x, r^{n} y\right)<\frac{r^{2 n} t}{5}$ for all $n \geq M_{1}$. Therefore by $\left(N_{5}\right)$ and $(2.7)$ we have

$$
N\left(D f\left(r^{n} x, r^{n} y\right), \frac{r^{2 n} t}{5}\right) \geq N\left(D f\left(r^{n} x, r^{n} y\right), t_{0} \varphi\left(r^{n} x, r^{n} y\right)\right) \geq 1-\varepsilon
$$

for all $n \geq M_{1}$. Thus $N(D Q(x, y), t) \geq 1-\varepsilon$ for all $x, y \in X$, all $t>0$ and all $0<\varepsilon<1$. It follows that $N(D Q(x, y), t)=1$ for all $t>0$, and $\left(N_{2}\right)$ implies that $D Q(x, y)=0$ for all $x, y \in X$. So $Q$ is quadratic by Proposition 2.1.

Now let for some positive $\delta$ and $\alpha,(2.4)$ holds. Let

$$
\varphi_{n}(x, 0):=\frac{1}{r^{2}} \sum_{k=0}^{n-1} \frac{\varphi\left(r^{k} x, 0\right)}{r^{2 k}}
$$

for all $x \in X \backslash\{0\}$. Let $x \in X \backslash\{0\}$ and $t>0$. By the same reasoning as in the beginning of the proof, similar to (2.10), one can deduce from (2.4) that

$$
N\left(\frac{f\left(r^{p} x\right)}{r^{2 p}}-f(x), \sum_{k=0}^{p-1} \frac{\delta}{r^{2 k+2}} \varphi\left(r^{k} x, 0\right)\right) \geq \alpha
$$

for all positive integers $p$. Hence we have

$$
\begin{aligned}
& N\left(f(x)-Q(x), \delta \varphi_{n}(x, 0)+t\right) \\
\geq & \min \left\{N\left(f(x)-\frac{f\left(r^{n} x\right)}{r^{2 n}}, \delta \varphi_{n}(x, 0)\right), N\left(\frac{f\left(r^{n} x\right)}{r^{2 n}}-Q(x), t\right)\right\} .
\end{aligned}
$$

Combining (2.11) and (2.12) and the fact that $\lim _{n \rightarrow \infty} N\left(\frac{f\left(r^{n} x\right)}{r^{2 n}}-Q(x), t\right)=1$, we observe that

$$
N\left(f(x)-Q(x), \delta \varphi_{n}(x, 0)+t\right) \geq \alpha
$$

for large enough $n \in \mathbb{N}$. It follows from the continuity of the real function $N(f(x)-Q(x), \cdot)$ that

$$
N\left(f(x)-Q(x), \frac{\delta}{r^{2}} \widetilde{\varphi}(x, 0)+t\right) \geq \alpha .
$$

Letting $t \rightarrow 0$, we conclude (2.5).

To end the proof, it remains to prove the uniqueness of $Q$. Let $T$ be another quadratic mapping satisfying (2.6). Given $\varepsilon>0$, by applying (2.6) for $Q$ and $T$, we can find some $t_{0}>0$ such that

$$
\begin{aligned}
& N(f(x)-Q(x), t \widetilde{\varphi}(x, 0)) \geq 1-\varepsilon, \\
& N(f(x)-T(x), t \widetilde{\varphi}(x, 0)) \geq 1-\varepsilon
\end{aligned}
$$


for all $x \in X \backslash\{0\}$ and all $t \geq t_{0}$. Fix some $x \in X \backslash\{0\}$ and $c>0$. So we find some integer $n_{0}$ such that

$$
t_{0} \sum_{k=n}^{\infty} r^{-2 k} \varphi\left(r^{k} x, 0\right)<\frac{c}{2}
$$

for all $n \geq n_{0}$. Since

$$
\sum_{k=n}^{\infty} r^{-2 k} \varphi\left(r^{k} x, 0\right)=\frac{1}{r^{2 n}} \sum_{k=0}^{\infty} r^{-2 k} \varphi\left(r^{n+k} x, 0\right)=\frac{1}{r^{2 n}} \widetilde{\varphi}\left(r^{n} x, 0\right),
$$

we have

$$
\begin{aligned}
& N(Q(x)-T(x), c) \\
\geq & \min \left\{N\left(\frac{f\left(r^{n} x\right)}{r^{2 n}}-Q(x), \frac{c}{2}\right), N\left(T(x)-\frac{f\left(r^{n} x\right)}{r^{2 n}}, \frac{c}{2}\right)\right\} \\
= & \min \left\{N\left(f\left(r^{n} x\right)-Q\left(r^{n} x\right), \frac{r^{2 n} c}{2}\right), N\left(T\left(r^{n} x\right)-f\left(r^{n} x\right), \frac{r^{2 n} c}{2}\right)\right\} \\
\geq & \min \left\{N\left(f\left(r^{n} x\right)-Q\left(r^{n} x\right), r^{2 n} t_{0} \sum_{k=n}^{\infty} r^{-2 k} \varphi\left(r^{k} x, 0\right)\right),\right. \\
& \left.N\left(T\left(r^{n} x\right)-f\left(r^{n} x\right), r^{2 n} t_{0} \sum_{k=n}^{\infty} r^{-2 k} \varphi\left(r^{k} x, 0\right)\right)\right\} \\
= & \min \left\{N\left(f\left(r^{n} x\right)-Q\left(r^{n} x\right), t_{0} \widetilde{\varphi}\left(r^{n} x, 0\right)\right), N\left(T\left(r^{n} x\right)-f\left(r^{n} x\right), t_{0} \widetilde{\varphi}\left(r^{n} x, 0\right)\right)\right\} \\
\geq & 1-\varepsilon .
\end{aligned}
$$

Therefore $N(Q(x)-T(x), c)=1$ for all $c>0$. Thus $Q(x)=T(x)$ for all $x \in X \backslash\{0\}$. Since $Q(0)=T(0)=0$, we have $Q=T$.

Corollary 2.3. Let $\theta, p, q>0$ and $\delta, \varepsilon$ be non-negative real numbers with $\delta^{2}+\varepsilon^{2} \neq 0$. Suppose that $f: \mathbb{X} \rightarrow Y$ is a function with $f(0)=0$ such that

$$
\lim _{t \rightarrow \infty} N(D f(x, y), t \varphi(x, y))=1
$$

uniformly on $\mathbb{X}^{2} \backslash\{(0,0)\}$, where $\varphi: \mathbb{X}^{2} \backslash\{(0,0)\} \rightarrow(0, \infty)$ is defined by

$$
\varphi(x, y)= \begin{cases}\delta+\varepsilon\left(\|x\|^{p}+\|y\|^{q}\right), & 0<p, q<2,|r|>1 ; \\ \theta\left(\|x\|^{p}+\|y\|^{q}\right), & p, q>2,|r|<1 .\end{cases}
$$

Then there is a unique quadratic mapping $Q: \mathbb{X} \rightarrow Y$ such that

$$
\lim _{t \rightarrow \infty} N(f(x)-Q(x), t \psi(x))=1
$$


uniformly on $\mathbb{X} \backslash\{0\}$, where

$$
\psi(x)= \begin{cases}\frac{\delta}{r^{2}-1}+\frac{\varepsilon}{r^{2}-|r|^{p}}\|x\|^{p}, & 0<p, q<2,|r|>1 ; \\ \frac{\|x\|^{p}}{r^{2}-|r|^{p}}, & p, q>2,|r|<1 .\end{cases}
$$

Remark 2.4. Let $f: X \rightarrow Y$ be a mapping with $f(0)=0$ for which there exists a function $\Phi: X^{2} \backslash\{(0,0)\} \rightarrow(0, \infty)$ such that

$$
\begin{gathered}
\widetilde{\Phi}(x, y):=\sum_{n=1}^{\infty} r^{2 n} \Phi\left(\frac{x}{r^{n}}, \frac{y}{r^{n}}\right)<\infty, \\
\lim _{t \rightarrow \infty} N(D f(x, y), t \Phi(x, y))=1
\end{gathered}
$$

uniformly on $X^{2} \backslash\{(0,0)\}$. By a similar method to the proof of Theorem 2.2, there exists a unique quadratic mapping $Q: X \rightarrow Y$ satisfying

$$
\lim _{t \rightarrow \infty} N(f(x)-Q(x), t \widetilde{\Phi}(x, 0))=1
$$

uniformly on $X \backslash\{0\}$. For the case

$$
\Phi(x, y)= \begin{cases}\delta+\varepsilon\left(\|x\|^{p}+\|y\|^{q}\right), & 0<p, q<2,|r|<1 ; \\ \theta\left(\|x\|^{p}+\|y\|^{q}\right), & p, q>2,|r|>1\end{cases}
$$

there exists a unique quadratic mapping $Q: \mathbb{X} \rightarrow Y$ satisfying

$$
Q(x):=N-\lim _{n \rightarrow \infty} r^{2 n} f\left(\frac{x}{r^{n}}\right), \quad \lim _{t \rightarrow \infty} N(f(x)-Q(x), t \Psi(x))=1
$$

uniformly on $\mathbb{X} \backslash\{0\}$, where

$$
\Psi(x)= \begin{cases}\frac{\delta}{1-r^{2}}+\frac{\varepsilon}{|r|^{p}-r^{2}}\|x\|^{p}, & 0<p, q<2,|r|<1 \\ \frac{\|x\|^{p}}{|r|^{p}-r^{2}}, & p, q>2,|r|>1 .\end{cases}
$$

Theorem 2.5. Let $\varphi: X^{2} \backslash\{(0,0)\} \rightarrow(0, \infty)$ be a function such that

$$
\widetilde{\varphi}(x, y):=\sum_{n=0}^{\infty} \frac{\varphi\left(2^{n} x, 2^{n} y\right)}{4^{n}}<\infty
$$

for all $(x, y) \in X^{2} \backslash\{(0,0)\}$. Let $f: X \rightarrow Y$ be an even mapping with $f(0)=0$ such that

$$
\lim _{t \rightarrow \infty} N(D f(x, y), t \varphi(x, y))=1
$$


uniformly on $X^{2} \backslash\{(0,0)\}$. Then $Q(x):=N$ - $\lim _{n \rightarrow \infty} \frac{f\left(2^{n} x\right)}{4^{n}}$ exists for each $x \in X$ and defines a quadratic mapping $Q: X \rightarrow Y$ such that if for some $\delta>0, \alpha>0$

$$
N\left(\Delta f(x, y), \delta \psi\left(\frac{x}{r}, \frac{y}{s}\right)\right) \geq \alpha
$$

for all $(x, y) \in(X \backslash\{0\}) \times(X \backslash\{0\})$, then

$$
N\left(f(x)-Q(x), \frac{\delta}{4} \sum_{k=0}^{\infty} \frac{1}{4^{k}} \psi\left(\frac{2^{k} x}{r}, \frac{2^{k} x}{s}\right)\right) \geq \alpha
$$

for all $x \in X \backslash\{0\}$, where

$$
\begin{aligned}
\psi(x, y) & :=\varphi(x, y)+\varphi(x,-y)+2 \varphi(x, 0)+2 \varphi(0, y), \\
\Delta f(x, y) & :=f(x+y)+f(x-y)-2 f(x)-2 f(y) .
\end{aligned}
$$

Furthermore, the quadratic mapping $Q: X \rightarrow Y$ is a unique mapping such that

$$
\lim _{t \rightarrow \infty} N\left(f(x)-Q(x), t \sum_{k=0}^{\infty} \frac{1}{4^{k}} \psi\left(\frac{2^{k} x}{r}, \frac{2^{k} x}{s}\right)\right)=1
$$

uniformly on $X \backslash\{0\}$.

Proof. It follows from (2.13) that

$$
\sum_{n=0}^{\infty} \frac{\psi\left(2^{n} x, 2^{n} y\right)}{4^{n}}<\infty
$$

for all $(x, y) \in(X \backslash\{0\}) \times(X \backslash\{0\})$. Since $f$ is even, it is clear that

$$
\begin{aligned}
\widehat{D} f(x, y): & =D f(x, y)+D f(x,-y)-2 D f(x, 0)-2 D f(0, y) \\
& =f(r x+s y)+f(r x-s y)-2 f(r x)-2 f(s y)
\end{aligned}
$$

for all $x, y \in X$. Since

$$
\begin{aligned}
& N(\widehat{D} f(x, y), t \psi(x, y)) \\
& \geq \min \{N(D f(x, y), t \varphi(x, y)), N(D f(x,-y), t \varphi(x,-y)), \\
&N(D f(x, 0), t \varphi(x, 0)), N(D f(0, y), t \varphi(0, y))\},
\end{aligned}
$$

it follows from (2.14) that

$$
\lim _{t \rightarrow \infty} N(\widehat{D} f(x, y), t \psi(x, y))=1
$$

uniformly on $(X \backslash\{0\}) \times(X \backslash\{0\})$. By (2.18), we deduce that

$$
\lim _{t \rightarrow \infty} N\left(\Delta f(x, y), t \psi\left(\frac{x}{r}, \frac{y}{s}\right)\right)=1
$$


uniformly on $(X \backslash\{0\}) \times(X \backslash\{0\})$. For a given $\varepsilon>0$, by (2.19), we can find some $t_{0}>0$ such that

$$
N\left(\Delta f(x, y), t \psi\left(\frac{x}{r}, \frac{y}{s}\right)\right) \geq 1-\varepsilon
$$

for all $(x, y) \in(X \backslash\{0\}) \times(X \backslash\{0\})$ and all $t \geq t_{0}$. Letting $y=x$ in (2.20), we get

$$
N\left(f(2 x)-4 f(x), t \psi\left(\frac{x}{r}, \frac{x}{s}\right)\right) \geq 1-\varepsilon
$$

for all $x \in X \backslash\{0\}$ and all $t \geq t_{0}$. Replacing $x$ by $2^{n} x$ in (2.21) and using $\left(N_{3}\right)$, we get

$$
N\left(\frac{f\left(2^{n+1} x\right)}{4^{n+1}}-\frac{f\left(2^{n} x\right)}{4^{n}}, \frac{t}{4^{n+1}} \psi\left(\frac{2^{n} x}{r}, \frac{2^{n} x}{s}\right)\right) \geq 1-\varepsilon
$$

for all $x \in X \backslash\{0\}$, all $t \geq t_{0}$ and all integers $n \geq 0$. Since

$$
\begin{aligned}
& N\left(\frac{f\left(2^{n+p} x\right)}{4^{n+p}}-\frac{f\left(2^{n} x\right)}{4^{n}}, \sum_{k=n}^{n+p-1} \frac{t}{4^{k+1}} \psi\left(\frac{2^{k} x}{r}, \frac{2^{k} x}{s}\right)\right) \\
= & N\left(\sum_{k=n}^{n+p-1}\left[\frac{f\left(2^{k+1} x\right)}{4^{k+1}}-\frac{f\left(2^{k} x\right)}{4^{k}}\right], \sum_{k=n}^{n+p-1} \frac{t}{4^{k+1}} \psi\left(\frac{2^{k} x}{r}, \frac{2^{k} x}{s}\right)\right) \\
\geq & \min _{n \leq k \leq n+p-1}\left\{N\left(\frac{f\left(2^{k+1} x\right)}{4^{k+1}}-\frac{f\left(2^{k} x\right)}{4^{k}}, \frac{t}{4^{k+1}} \psi\left(\frac{2^{k} x}{r}, \frac{2^{k} x}{s}\right)\right)\right\},
\end{aligned}
$$

we get from (2.22) that

$$
N\left(\frac{f\left(2^{n+p} x\right)}{4^{n+p}}-\frac{f\left(2^{n} x\right)}{4^{n}}, \sum_{k=n}^{n+p-1} \frac{t_{0}}{4^{k+1}} \psi\left(\frac{2^{k} x}{r}, \frac{2^{k} x}{s}\right)\right) \geq 1-\varepsilon
$$

for all $x \in X \backslash\{0\}$ and all integers $n \geq 0, p \geq 1$. Similar to the proof of Theorem 2.2 , we conclude from (2.23) that the sequence $\left\{\frac{f\left(2^{n} x\right)}{4^{n}}\right\}$ is Cauchy in $Y$ for each $x \in X$. So we can define a (quadratic) mapping $Q: X \rightarrow Y$ by $Q(x):=$ $N-\lim _{n \rightarrow \infty} \frac{f\left(2^{n} x\right)}{4^{n}}$, namely, for each $t>0$ and $x \in X, \lim _{n \rightarrow \infty} N\left(\frac{f\left(2^{n} x\right)}{4^{n}}-\right.$ $Q(x), t)=1$. The rest of the proof is similar to the proof of Theorem 2.2 and we omit the details.

Corollary 2.6. Let $0<p, q<2$ and $\delta$, $\varepsilon$ be real numbers with $\delta^{2}+\varepsilon^{2} \neq 0$. Suppose that $f: \mathbb{X} \rightarrow Y$ is an even function with $f(0)=0$ such that

$$
\lim _{t \rightarrow \infty} N(D f(x, y), t \varphi(x, y))=1
$$

uniformly on $\mathbb{X}^{2} \backslash\{(0,0)\}$, where $\varphi: \mathbb{X}^{2} \backslash\{(0,0)\} \rightarrow(0, \infty)$ is defined by

$$
\varphi(x, y):=\delta+\varepsilon\left(\|x\|^{p}+\|y\|^{q}\right) .
$$

Then there is a unique quadratic mapping $Q: \mathbb{X} \rightarrow Y$ such that

$$
\lim _{t \rightarrow \infty} N(f(x)-Q(x), t \psi(x))=1
$$


uniformly on $\mathbb{X} \backslash\{0\}$, where

$$
\psi(x)=\delta+\frac{2 \varepsilon}{|r|^{p}\left(4-2^{p}\right)}\|x\|^{p}+\frac{2 \varepsilon}{|s|^{q}\left(4-2^{q}\right)}\|x\|^{q} .
$$

Remark 2.7. Let $f: X \rightarrow Y$ be an even mapping with $f(0)=0$ for which there exists a function $\Phi: X^{2} \backslash\{(0,0)\} \rightarrow(0, \infty)$ such that

$$
\begin{gathered}
\widetilde{\Phi}(x, y):=\sum_{n=1}^{\infty} 4^{n} \Phi\left(\frac{x}{2^{n}}, \frac{y}{2^{n}}\right)<\infty, \\
\lim _{t \rightarrow \infty} N(D f(x, y), t \Phi(x, y))=1
\end{gathered}
$$

uniformly on $X^{2} \backslash\{(0,0)\}$. By a similar method to the proof of Theorem 2.5, there exists a unique quadratic mapping $Q: X \rightarrow Y$ satisfying

$$
\lim _{t \rightarrow \infty} N\left(f(x)-Q(x), t \sum_{k=1}^{\infty} 4^{k} \psi\left(\frac{x}{2^{k} r}, \frac{x}{2^{k} s}\right)\right)=1
$$

uniformly on $X \backslash\{0\}$, where $\psi(x, y):=\varphi(x, y)+\varphi(x,-y)+2 \varphi(x, 0)+2 \varphi(0, y)$. For the case

$$
\Phi(x, y)=\varepsilon\left(\|x\|^{p}+\|y\|^{q}\right), \quad(p, q>2, \varepsilon>0)
$$

there exists a unique quadratic mapping $Q: \mathbb{X} \rightarrow Y$ satisfying

$$
Q(x):=N-\lim _{n \rightarrow \infty} 4^{n} f\left(\frac{x}{2^{n}}\right), \quad \lim _{t \rightarrow \infty} N(f(x)-Q(x), t \Psi(x))=1
$$

uniformly on $\mathbb{X} \backslash\{0\}$, where

$$
\Psi(x)=\frac{\|x\|^{p}}{|r|^{p}\left(2^{p}-4\right)}+\frac{\|x\|^{q}}{|s|^{q}\left(2^{q}-4\right)} .
$$

For the case $p=q=2$, we have the following counterexample.

Example 2.8. Let $\phi: \mathbb{R} \rightarrow \mathbb{R}$ be defined by

$$
\phi(x):= \begin{cases}\mu x^{2} & \text { for }|x|<1 \\ \mu & \text { for }|x| \geq 1\end{cases}
$$

where $\mu$ is a positive real number. Consider the function $f: \mathbb{R} \rightarrow \mathbb{R}$ by the formula

$$
f(x):=\sum_{n=0}^{\infty} \alpha^{-2 n} \phi\left(\alpha^{n} x\right),
$$

where $\alpha=\sqrt{1+r^{2}+s^{2}+|r s|}$. Then $f$ is continuous, bounded and satisfies

$$
|D f(x, y)| \leq \frac{\alpha^{10}}{\alpha^{2}-1} \mu\left(x^{2}+y^{2}\right)
$$

for all $x, y \in \mathbb{R}$ (see [21]). So

$$
\lim _{t \rightarrow \infty} N\left(D f(x, y), t\left(x^{2}+y^{2}\right)\right)=1
$$


uniformly on $\mathbb{R}^{2} \backslash\{(0,0)\}$, where $N(\cdot, \cdot)$ is the fuzzy norm on $\mathbb{R}$ defined in Example 1.6. Let $Q: \mathbb{R} \rightarrow \mathbb{R}$ be a quadratic function such that

$$
\lim _{t \rightarrow \infty} N\left(f(x)-Q(x), t x^{2}\right)=1
$$

uniformly on $\mathbb{R} \backslash\{0\}$. Hence there exists a constant $\beta>0$ such that $\mid f(x)-$ $Q(x) \mid \leq \beta x^{2}$ for all $x \in \mathbb{R}$. Since $Q$ is quadratic, there exists a constant $c \in \mathbb{R}$ such that $Q(x)=c x^{2}$ for all rational numbers $x$. Therefore

$$
|f(x)| \leq(\beta+|c|) x^{2}
$$

for all rational numbers $x$. Let $m$ be an integer with $m \mu>\beta+|c|$. If $x$ is a rational number in $\left(0, \alpha^{1-m}\right)$, then $\alpha^{n} x \in(0,1)$ for all $n=0,1, \ldots, m-1$. So

$$
f(x) \geq \sum_{n=0}^{m-1} \alpha^{-2 n} \phi\left(\alpha^{n} x\right)=m \mu x^{2}>(\beta+|c|) x^{2}
$$

which contradicts $(2.24)$

Acknowledgments. The author would like to thank the referee for a number of valuable suggestions regarding a previous version of this paper.

\section{References}

[1] J. Aczél and J. Dhombres, Functional Equations in Several Variables, Cambridge University Press, Cambridge, 1989.

[2] D. Amir, Characterizations of Inner Product Spaces, Birkhäuser, Basel, 1986.

[3] T. Aoki, On the stability of the linear transformation in Banach spaces, J. Math. Soc. Japan 2 (1950), 64-66.

[4] T. Bag and S. K. Samanta, Finite dimensional fuzzy normed linear spaces, J. Fuzzy Math. 11 (2003), no. 3, 687-705.

[5] _ Fuzzy bounded linear operators, Fuzzy Sets and Systems 151 (2005), no. 3, 513-547.

[6] P. W. Cholewa, Remarks on the stability of functional equations, Aequationes Math. 27 (1984), no. 1-2, 76-86.

[7] S. Czerwik, On the stability of the quadratic mapping in normed spaces, Abh. Math. Sem. Univ. Hamburg 62 (1992), 59-64.

8] _ Functional Equations and Inequalities in Several Variables, World Scientific Publishing Company, New Jersey, Hong Kong, Singapore and London, 2002.

[9] P. Găvruţa, A generalization of the Hyers-Ulam-Rassias stability of approximately additive mappings, J. Math. Anal. Appl. 184 (1994), no. 3, 431-436.

[10] A. Grabiec, The generalized Hyers-Ulam stability of a class of functional equations, Publ. Math. Debrecen 48 (1996), no. 3-4, 217-235.

[11] D. H. Hyers, On the stability of the linear functional equation, Proc. Nat. Acad. Sci. U.S.A. 27 (1941), 222-224.

[12] D. H. Hyers, G. Isac, and Th. M. Rassias, Stability of Functional Equations in Several Variables, Birkhäuser, Basel, 1998.

[13] P. Jordan and J. von Neumann, On inner products in linear, metric spaces, Ann. of Math. (2) 36 (1935), no. 3, 719-723.

[14] K. Jun and Y. Lee, On the Hyers-Ulam-Rassias stability of a Pexiderized quadratic inequality, Math. Inequal. Appl. 4 (2001), no. 1, 93-118.

[15] S.-M. Jung, Hyers-Ulam-Rassias Stability of Functional Equations in Mathematical Analysis, Hadronic Press, Inc., Palm Harbor, FL, 2001. 
[16] Pl. Kannappan, Quadratic functional equation and inner product spaces, Results Math. 27 (1995), no. 3-4, 368-372.

[17] D. Mihet and V. Radu, On the stability of the additive Cauchy functional equation in random normed spaces, J. Math. Anal. Appl. 343 (2008), no. 1, 567-572.

[18] A. K. Mirmostafaee, M. Mirzavaziri, and M. S. Moslehian, Fuzzy stability of the Jensen functional equation, Fuzzy Sets and Systems 159 (2008), no. 6, 730-738.

[19] A. K. Mirmostafee and M. S. Moslehian, Fuzzy versions of Hyers-Ulam-Rassias theorem, Fuzzy Sets and Systems 159 (2008), no. 6, 720-729.

[20] _ Fuzzy approximately cubic mappings, Inform. Sci. 178 (2008), no. 19, 37913798.

[21] A. Najati and C. Park, Fixed points and stability of a generalized quadratic functional equation, J. Inequal. Appl. 2009 (2009), Article ID 193035, 19 pages.

[22] C. Park, Fuzzy stability of a functional equation associated with inner product spaces, Fuzzy Sets and Systems 160 (2009), no. 11, 1632-1642.

[23] _ On the stability of the linear mapping in Banach modules, J. Math. Anal. Appl. 275 (2002), no. 2, 711-720.

[24] Th. M. Rassias, On the stability of the linear mapping in Banach spaces, Proc. Amer. Math. Soc. 72 (1978), no. 2, 297-300.

[25] - Functional Equations, Inequalities and Applications, Kluwer Academic Publishers Co., Dordrecht, Boston, London, 2003.

[26] F. Skof, Local properties and approximation of operators, Rend. Sem. Mat. Fis. Milano 53 (1983), 113-129.

[27] S. M. Ulam, A Collection of the Mathematical Problems, Interscience Publ. New York, 1960.

Department of Mathematics

University of Mohaghegh Ardabili

ARDABIL 56199-11367, IRAN

E-mail address: a.nejati@yahoo.com 